\title{
Grado de Influencia de la Aptitud para Aprender el Idioma Inglés y el Nivel de Conocimientos Previos en el Rendimiento Académico de los Alumnos de la Especialidad de Idioma Extranjero, Traductor e Intérprete de la FECH de la Universidad Nacional Jorge Basadre Grohmann de Tacna, 2011
}

\author{
Aptitude Influence Degree to learn English and the Previous Knowledge \\ Level in the Academic Results of the Students of Foreign Language, \\ Translator and Interpreter of the Faculty of Education, Comunication \\ and Humanities in Jorge Basadre Grohmann University of Tacna, 2011
}

\author{
${ }^{1}$ Cecilia Mendoza Gómez, ${ }^{2}$ Silvia Bazán Velásquez
}

\begin{abstract}
RESUMEN:
En la presente investigación participaron todos los estudiantes ingresantes al primer año y matriculados en la especialidad de Idioma Extranjero, Traductor e Intérprete. El motivo de la investigación radica en que durante el proceso de enseñanza-aprendizaje del idioma inglés, se observa que los alumnos presentan dificultad en el aprendizaje del idioma inglés, esto reflejado en su deficiente captación de los sonidos, inapropiada entonación y deficiente acentuación; posiblemente estos problemas surgen debido a que al ingresar no se les aplica un test de aptitud para el aprendizaje de un idioma extranjero y al uso inadecuado de material didáctico incluidos los medios audio visuales que les permita una mejor captación del idioma. Debido a esto se trató de evidenciar la relación entre la Aptitud para aprender un idioma extranjero y el rendimiento académico. Para tal efecto, se aplicó un test de Aptitud especificamente relacionado al aprendizaje del idioma inglés, se hizo una observación de los registros académicos durante el año académico y finalmente se determinó el nivel de significancia entre ambas variables.

El presente trabajo de investigación se justificó en su valor teórico, utilidad práctica, relevancia académica, por su conveniencia yen base a los beneficios netos que genera.
\end{abstract}

Palabras Clave: Aptitud, Entonación, Acentuación, Test de Aptitud, Rendimiento Académico

\section{ABSTRACT:}

All the students of the first year enrolled in the specialty of Foreign Language, Translator and Interpreter participated in this research. The purpose of the investigation is that during the process of teaching and learning English, it appears that students have difficulty in learning the English language, this reflected in their poor uptake of sounds, poor intonation and inappropriate stress, possibly these problems arise because at the entrance the students are not given an aptitude test for learning a foreign language and the inappropriate use of teaching materials including audio visual media that enables them to an increasing awareness of language. Because of this it was evident the relationship between the ability to learn a foreign language and the academic performance. To this end, we applied an aptitude test specifically related to English language learning, an observation of academic records during the academic year was made and finally we determined the level of significance between the two variables.

The present investigation was justified in its theoretical value, practical and academic relevance, for its convenience and based on the net profit that it will generate.

Keywords: Aptitude, Intonation, Stress, Aptitude Test, Academic Performance.

\footnotetext{
' Lic. en Educación, Especialidad Idiomas Inglés - Francés. Facultad de Educación, Comunicación y Humanidades de la Universidad Nacional Jorge Basadre Grohmann.

${ }^{2}$ Magister en Docencia Universitaria y Gestión Educativa. Lic. en Educación, Especialidad de Idiomas Inglés / Alemán. Facultad de Educación, Comunicación y Humanidades, Universidad Nacional Jorge Basadre Grohmann.
} 


\section{I.- INTRODUCCIÓN}

En el presente siglo, la ciencia y la tecnología hacen que el mundo avance, se realizan importantes transformaciones económicas y sociales, los medios de comunicación han tenido un gran desarrollo, los movimientos migratorios y de sus relativos intercambios entre culturas nos enseña que evolucionamos y nos enriquecemos a través de ellos, de ello surge la necesidad de apropiarnos de una lengua extranjera que fomente nuestras posibilidades de comunicación oral y escrita y dentro de dicha comunicación el inglés juega el papel principal que propicia la globalización comunicativa.

Diversos autores opinan que la mejor forma de aprendizaje del idioma inglés se logra si es que el estudiante tiene aptitud para aprender un idioma extranjero (inglés) y que ésto influye en el rendimiento académico y la satisfacción personal de estudiante. El aprendizaje de un idioma extranjero como el inglés, representa para muchos estudiantes, de todo nivel educativo, serias dificultades.

Es urgente revisar los paradigmas curriculares en los cuales se sustenta la enseñanza del idioma inglés, sobre todo en países menos desarrollados, teniendo en cuenta que el inglés - como capacidad comunicativa - muchas veces se describe en términos de cuatro habilidades: producción oral y producción escrita (habilidades productivas) y, comprensión auditiva y comprensión lectora (habilidades receptivas).

El objetivo de este trabajo es determinar la influencia de la Aptitud para el aprendizaje de un idioma extranjero y el Rendimiento Académico de los alumnos de la Carrera Profesional de Educación en la especialidad de Idioma Extranjero, Traductor e Intérprete de la Universidad Nacional Jorge Basadre Grohmann, correspondiente al primer año de estudios, y contribuir al mejoramiento de la selección de los estudiantes participantes de la especialidad de IETI y al desarrollo académico.

\section{Objetivos}

Evaluar la Aptitud para aprender el idioma Inglés de los estudiantes.

Evaluar el Rendimiento Académico de los estudiantes

Determinar la Relación entre la Aptitud para aprender un idioma extranjero y el Rendimiento Académico de los estudiantes de la especialidad de Idioma Extranjero Traductor e Intérprete de la FECH de la UNJBG de Tacna.

\section{II.- MATERIALESY MÉTODOS}

\subsection{Ubicación}

El trabajo se realizó en la Facultad de Educación, Comunicaciones y Humanidades, en la escuela académico profesional de Educación.

\subsection{Muestra}

La población meta estuvo constituida por 200 alumnos matriculados de 1 ro a 5 to año en la especialidad de Ieti y la población muestra por 60 alumnos matriculados en el ler año a quienes se les aplicó el test de aptitud al inicio del año académico y se les hizo el seguimiento académico respectivo durante el año académico 2011.

\subsection{Metodología}

La técnica de recolección de datos se realizó de la siguiente manera :

1.- Aplicación de un Test de Aptitud para el aprendizaje de idiomas extranjeros el cual sirvió para diagnosticar qué grupo de alumnos poseía cierto grado de aptitud.

2.- Observación del proceso de aprendizaje cuyo resultado se plasmaba en el Rendimiento Académico.

3.- Comparación del grupo con cierto grado de aptitud para el aprendizaje de inglés con el grupo sin aptitud para el aprendizaje de inglés y el resultado académico durante el año académico lectivo 2011.

\section{III.- RESULTADOS}

El producto de la experiencia comprende la presentación, análisis e interpretación de los resultados de la aplicación del test de Aptitud para el aprendizaje de un idioma extranjero y los resultados académicos.

Comprende tres fases:

1.- La aplicación del test de Aptitud al grupo de estudio al inicio del año académico.

2.- Observación y estudio de los resultados del rendimiento académico de dicho grupo de estudio.

3.- Establecer las comparaciones entre el grupo con cierto grado de aptitud para el aprendizaje de un idioma extranjero y el grupo sin aptitud para el aprendizaje de un idioma.

4.- Elaboración de cuadros estadístico para una mejor interpretación de resultados.

\subsection{Análisis de los Resultados}

Tabla No. 1

Universidad Nacional Jorge Basadre Grohmann Grado de Aptitud para el Aprendizaje de un Idioma Extranjero

\begin{tabular}{|c|c|c|c|}
\hline GRADO & Frecuencia & Porcentaje & $\begin{array}{c}\text { Porcentaje } \\
\text { acumulado }\end{array}$ \\
\hline BAJO & 42 & 70,0 & 70,0 \\
ALTO & 18 & 30,0 & 30,0 \\
Total & 60 & 100,0 & 100,0 \\
\hline
\end{tabular}

Fuente: Test de Aptitud para el aprendizaje de un idioma extranjero 
Universidad Nacional Jorge Basadre Grohamann Grado de Aptitud para el Aprendizaje de un Idioma Extranjero

Estudiantes del ler año de IETI

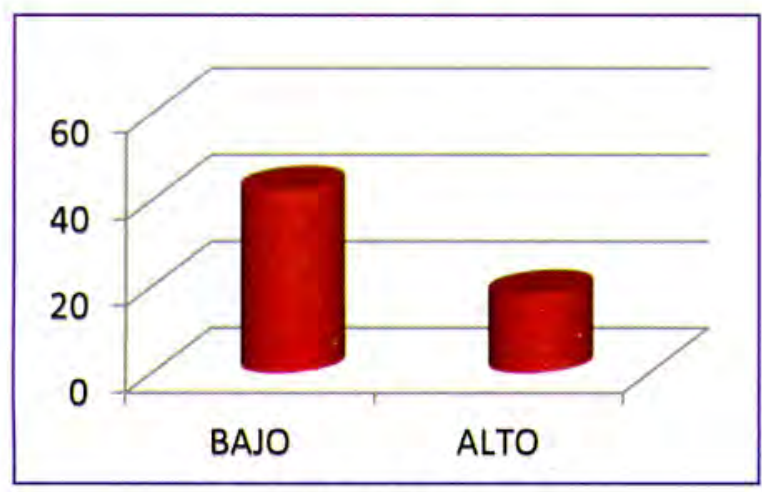

Figura No. 1

\section{IV.-DISCUSIÓN}

De acuerdo a los datos de la Tabla Nro. 1, que corresponde a la tabulación de los resultados obtenidos por el Grupo en Estudio en el test de aptitud para aprender un idioma extranjero, se evidencia que el $70 \%$ de los estudiantes ingresantes al primer año de la especialidad de Idioma Extranjero, traductor e Intérprete no tiene aptitud para aprender un idioma. El $30 \%$ restante sí tiene aptitud para el aprendizaje de un idioma extranjero.

Tomando en cuenta los ítems que conforman el test de aptitud, esto quiere decir que los estudiantes que conforman el $70 \%$ muestran aptitud para aprender un idioma extranjero de forma muy escasa o nula, no existe retención ni dominio del idioma. Consecuentemente, el rendimiento es muy bajo.

Para efectos de que el presente trabajo de investigación cumpla su cometido se recomienda lo siguiente:

1.- El test de Aptitud para aprender un idioma extranjero nos demuestra ser imprescindible para la selección de estudiantes que postulan a la especialidad de Idioma Extranjero, traductor e intérprete, por ello se sugiere aplicar dicho test a los postulantes para el mejor proceso de enseñanza-aprendizaje.

2.- Además del test de Aptitud para el aprendizaje de un idioma extranjero, se sugiere tomar una prueba de conocimientos básico-intermedio del idioma inglés para que así las sesiones de clases se realicen exclusivamente en el idioma meta (inglés) y de esta manera evitar los grupos heterogéneos en el primer año de estudios

\section{V.- CONCLUSIONES}

\section{Primera}

Los resultados de la investigación permitieron determinar la relación directa entre la Aptitud para aprender un idioma extranjero y el rendimiento académico de los estudiantes de 1er año de la especialidad de Idioma Extranjero, Traductor e Intérprete de la Escuela Profesional de Educación de la Universidad Nacional Jorge Basadre Grohmann de Tacna en el año 2011.

\section{Segunda}

Los estudiantes que demuestran tener aptitud para aprender un idioma extranjero, tienen mejor nivel de dominio y retención del idioma en estudio (inglés).

\section{Tercera}

Los estudiantes que no tienen aptitud para aprender un idioma extranjero, tienen bajo rendimiento académico.

\section{REFERENCIAS BIBLIOGRÁFICAS}

Alcaraz,E. y Moody,E. (1982): Didáctica del inglés: Metodología y programación. Madrid: Alhambra Universidad.

Alcaraz, E. y Ramón,J.(1980):La evaluación del inglés. Teoría y práctica. SGEL, Madrid.

Alderson, C.(ed.)(1985): Evaluation (Lancaster Practical Papers in English Language Education, Vol.6). Pergamon, Oxford.

Alderson, J.C. \& Hughes,(eds.)(1981): ELT Documents III: Issues in Language Testing. British Council.

Alpert,R., y Haber, R.(1960):"Anxiety in academic achievement situations". Journal of Abnormal and Social Psychology, 61:297-215.

Andersen, R.,(1979):"The relationship between first language transfer and second language overgeneralization: data from the English of Spanish speakers". En Andersen, R.(ed.). The acquisition and use of Spanish and English as first and second languages, Washington, D.C.: TESOL, pp. 43-58.

Bayley K.M.,(1980): "An introspective analysis of an individual's language learning experience". En Krasen,S. y Scarcella, R.(eds.). Research in second language acquisition, Newbury House. Rowley, Mass.

Bruner,J.S.(1978): El proceso mental en el aprendizaje. Narcea, Madrid.

Carrol,J.B. y Sapon,S.M.(1959): Modern Language Aptitude Test.(MLAT). New York: Psychological Corporation. 68,n1 4.

Carrol, J.B. (1967): Modern Language Aptitude TestElementary.( EMLAT). New York, Psychological Corporation. (369-73).

Dagut,M. y Laufer,B.(1985):oidance of phrasal verbs: a case for contrastive analysis". Studies in Second Language Acquisition, 7: 73-79.

Fernandez Huerta, J.(1973):"Conceptos fundamentales. 
La didáctica y Enseñanza y aprendizaje". En Maillo (dir.): Enciclopedia de la didáctica aplicada. Labor, pp.10-72, Madrid.

Fries, C.,(1945): Teaching and Learning English as a Foreign Language, Ann Arbor: University of Michigan Press.

Gass,S., (eds.)(1989): Linguistic perspectives on second language acquisition, New York: Cambridge University Press

I.C.E. (1986...):"Aspectos Didácticos del inglés. N1s. de 1 a 5. Educación Abierta. Zaragoza.

Larsen-Freeman, D. y M.H.Long,(1991): An Introduction to Second Language Acquisition Research. Longman, Londres.
Major, R. (1987):"Phonological similarity, markedness, and rate of L2 acquisition". Studies in Second Language Acquisition, 9: 63-82.

Naiman, N., Frolich,M., Stern, H,y Todesco, A (1978): The good language learner. Research in Education Series n1.7, The Ontario Institute for Studies in Education, Toronto.

\section{Correspondencia:}

Lic. Cecilia Mendoza Gómez

Ciudad Universitaria fundo "Los Granados"

Av. Miraflores s/n Tacna. Perú.

Ceci1966@gmail.com 